\title{
Accurate Antenna Models in Ground Penetrating Radar Diffraction Tomography
}

\author{
Meincke, Peter; Kim, Oleksiy S.
}

Published in:

Proceedings of IEEE Antennas and Propagation Society International Symposium

Link to article, DOI:

10.1109/APS.2002.1016984

Publication date:

2002

Document Version

Publisher's PDF, also known as Version of record

Link back to DTU Orbit

Citation $(A P A)$ :

Meincke, P., \& Kim, O. S. (2002). Accurate Antenna Models in Ground Penetrating Radar Diffraction

Tomography. In Proceedings of IEEE Antennas and Propagation Society International Symposium (Vol. 4, pp. 306-309). IEEE. https://doi.org/10.1109/APS.2002.1016984

\section{General rights}

Copyright and moral rights for the publications made accessible in the public portal are retained by the authors and/or other copyright owners and it is a condition of accessing publications that users recognise and abide by the legal requirements associated with these rights.

- Users may download and print one copy of any publication from the public portal for the purpose of private study or research.

- You may not further distribute the material or use it for any profit-making activity or commercial gain

- You may freely distribute the URL identifying the publication in the public portal 


\section{Accurate Antenna Models in Ground Penetrating Radar Diffraction Tomography}

Peter Meincke and Oleksiy Kim*

Ørsted-DTU, Section for Electromagnetic Systems, Building 348, Technical University of Denmark, DK-2800 Kgs. Lyngby, Denmark, pme@oersted.dtu.dk.

\section{Introduction}

Linear inversion schemes based upon the concept of diffraction tomography have proven successful for ground penetrating radar (GPR) imaging [1], [2], [3]. In many GPR surveys, the antennas of the GPR are located close to the air-soil interface and therefore, it is important, as illustrated in [2], to incorporate in the inversion scheme the presence of this interface. In [2] the antennas are modeled as ideal (Hertzian) electric dipoles. Since practical GPR antennas are not ideal, it is of interest to investigate the validity of this model.

In this paper we extend the formulation of [2] to hold for arbitrary antennas. For simplicity the 2.5-D case is considered, that is, it is assumed that the scattering object in the soil is invariant in one direction, which for instance is the case for a pipe. The arbitrary antennas are modeled by their plane-wave receiving- and transmitting spectra. We find these: spectra numerically for a resistively loaded dipole using the method of moments. Also, we illustrate through a numerical example the importance of taking into account the correct antenna pattern in GPR diffraction tomography.

\section{The 2.5-D Forward Model}

An example of the GPR configuration involving the planar air-soil interface is shown in Figure 1. A Cartesian $x y z$ coordinate system is introduced such that the $x y$ plane coincides with the interface and such that $z>0$ is air. An object, which is assumed infinitely long in the $\hat{\mathbf{x}}$ direction, is buried in the soil. The propagation constant of air is $k_{0}(\omega)=\omega \sqrt{\mu_{0} \epsilon_{0}}$ and that of soil is $k_{1}(\omega)=\omega \sqrt{\mu_{0} \epsilon_{1}}$, assuming that the soil is lossless. The position of the receiving antenna is described by $\left(x_{r}, y_{r}, z_{r}\right)$ and that of the transmitting antenna is $\left(x_{t}, y_{t}, z_{t}\right)=\left(x_{r}+x_{\Delta}, y_{r}+y_{\Delta}, z_{r}\right)$ with the offset $\left(x_{\Delta}, y_{\Delta}, 0\right)$ being fixed. It is assumed that the conductivity $\sigma(y, z)$ is much less than the contrast in permittivity $\Delta \epsilon(y, z)=\epsilon(y, z)-\epsilon_{1}$, i.e., $\sigma(y, z) \ll \omega \Delta \epsilon(y, z)$ over the frequency band of interest. Assuming an $\hat{\mathbf{x}}$-directed antenna described by the current density $J_{b}(x, y, z, \omega)$, the background electric field $\mathbf{E}_{b}$ in the soil can be expressed as

$$
\begin{aligned}
\mathbf{E}_{b}(x, y, z, \omega) & =\frac{1}{(2 \pi)^{2}} \int_{-\infty}^{\infty} \int_{\mathbf{S}_{b}\left(k_{x}, k_{y}, \omega\right) \exp \left(i \left[k_{x}\left(x-x_{t}\right)+k_{y}\left(y-y_{t}\right)\right.\right.} \\
& \left.\left.\left.+\gamma_{0} z_{t}-\gamma_{1} z\right]\right) d k_{x} d k_{y} \quad \text { (time factor } \exp (-i \omega t)\right)
\end{aligned}
$$

where the plane-wave transmitting spectrum $\mathbf{S}_{b}$ is $[3,(4)]$

$$
\mathbf{S}_{b}\left(k_{x}, k_{y}, \omega\right)=\frac{-\omega \mu_{0}}{2} \tilde{J}_{b}\left(k_{x}, k_{y},-\gamma_{0}, \omega\right) \hat{\mathbf{x}} \cdot \tilde{\mathbf{F}}\left(-k_{x},-k_{y}, \omega\right) .
$$

*The Danish Technical Research Council is acknowledged for supporting this work. 
Herein, $\gamma_{i}=\gamma_{i}\left(k_{x}, k_{y}, \omega\right)=\sqrt{k_{i}^{2}(\omega)-k_{x}^{2}-k_{y}^{2}}, i=0,1$, the expression for the dyadic $\overline{\mathbf{F}}\left(k_{x}, k_{y}, \omega\right)$ describing the interface can be found in $[2,(6)]$, and $\tilde{J}_{b}$ is the Fourier transform of the current density defined as

$$
\tilde{J}_{b}\left(k_{x}, k_{y}, k_{z}, \omega\right)=\iint_{-\infty}^{\infty} \int_{b} J_{b}(x, y, z, \omega) \exp \left(-i\left[k_{x} x+k_{y} y+k_{z} z\right]\right) d x d y d z .
$$

Assuming that the buried object is a weak scatterer, such that it satisfies the Born approximation, the output $s$ of the receiving antenna is

$$
\begin{aligned}
s\left(x_{r}, y_{r}, z_{r}, \omega\right) & =\frac{i \omega^{2} \mu_{0}}{8 \pi^{2}} \iint_{-\infty}^{\infty} \frac{1}{\gamma_{1}} \mathbf{R}\left(k_{x}, k_{y}, \omega\right) \cdot \iiint_{z^{\prime} \leq 0} \mathbf{E}_{b}\left(x^{\prime}, y^{\prime}, z^{\prime}, \omega\right) \Delta \epsilon\left(y^{\prime}, z^{\prime}\right) \\
& \cdot \exp \left(i\left[k_{x}\left(x_{r}-x^{\prime}\right)+k_{y}\left(y_{r}-y^{\prime}\right)+\gamma_{0} z_{\tau}-\gamma_{1} z^{\prime}\right]\right) d x^{\prime} d y^{\prime} d z^{\prime} d k_{x} d k_{y} \text { (4) }
\end{aligned}
$$

where $\mathbf{R}$ is the receiving plane-wave spectrum of the antenna. In case of a reciprocal antenna, the receiving spectrum is related to the nonmalized transmitting spectrum $\mathbf{S}$ (resulting from an input signal of 1 volt) as

$$
\mathbf{R}\left(k_{x}, k_{y}, \omega\right)=\frac{\gamma_{1}\left(k_{x}, k_{y}, \omega\right)}{\omega \mu_{0} Y_{0}} \mathbf{S}\left(-k_{x},-k_{y}, \omega\right)
$$

where $Y_{0}$ is the characteristic admittance of the transmission line connected to the receiving antenna. Replacing $\left(k_{x}, k_{y}\right)$ with $\left(k_{x}^{\prime}, k_{y}^{\prime}\right)$ in the expression (1) for the background field and inserting into (4), performing the integration over $x^{\prime}$ and $k_{x}$, carrying out the substitution $k_{y}=k_{y}+k_{y}^{\prime}$, and Fourier transforming with respect to $y_{r}$, we arrive at

$$
\begin{aligned}
& \tilde{s}\left(k_{y}, z_{r}, \omega\right)=\frac{i \omega^{2} \mu_{0}}{8 \pi^{2}} \int_{-\infty}^{\infty} \frac{\mathbf{R}\left(k_{x}^{\prime}, k_{y}+k_{y}^{\prime}, \omega\right) \cdot \mathbf{S}_{b}\left(k_{x}^{\prime}, k_{y}^{\prime}, \omega\right)}{\gamma_{1}\left(k_{x}^{\prime}, k_{y}+k_{y}^{\prime}, \omega\right)} \\
& \quad \cdot \exp \left(i\left[\left(\gamma_{0}\left(k_{x}^{\prime}, k_{y}^{\prime}, \omega\right)+\gamma_{0}\left(k_{x}^{\prime}, k_{y}+k_{y}^{\prime}, \omega\right)\right) z_{r}-k_{x}^{\prime} x_{\Delta}-k_{y}^{\prime} y_{\Delta}\right]\right) \\
& \quad \cdot \iint_{z^{\prime} \leq 0} \Delta \epsilon\left(y^{\prime}, z^{\prime}\right) \exp \left(-i\left[k_{y} y^{\prime}+\left(\gamma_{1}\left(k_{x}^{\prime}, k_{y}^{\prime}, \omega\right)+\gamma_{1}\left(k_{x}^{\prime}, k_{y}+k_{y}^{\prime}, \omega\right)\right) z^{\prime}\right]\right) d y^{\prime} d z^{\prime} d k_{x}^{\prime} d k_{y}^{\prime} .
\end{aligned}
$$

Now we can use the result of [2, Appendix] to calculate the integration over $k_{x}^{\prime}$ and $k_{y}^{\prime}$ asymptotically as $z^{\prime} \rightarrow-\infty$. The result is

$$
\tilde{s}\left(k_{y}, z_{r}, \omega\right) \sim D\left(k_{y}, \omega\right) \widetilde{\Delta \epsilon}_{1}\left(k_{y}, \sqrt{4 k_{1}^{2}(\omega)-k_{y}^{2}}\right)
$$

where

$$
\begin{aligned}
& D\left(k_{y}, \omega\right)=\frac{-\omega^{2} \mu_{0}}{16 \pi k_{1}(\omega)} \mathbf{R}\left(0, \frac{1}{2} k_{y}, \omega\right) \cdot \mathbf{S}_{b}\left(0,-\frac{1}{2} k_{y}, \omega\right) \sqrt{4 k_{1}^{2}(\omega)-k_{y}^{2}} \\
& \cdot \exp \left(i\left[\sqrt{4 k_{0}^{2}(\omega)-k_{y}^{2} z_{\tau}}+\frac{1}{2} k_{y} y_{\Delta}\right]\right)
\end{aligned}
$$

and $\Delta \epsilon_{1}(y, z)=\Delta \epsilon(y, z) / z$. Using (5), (2), and [2, (6)], it can be shown that $\mathbf{R}\left(0, \frac{1}{2} k_{y}, \omega\right)$. $\mathbf{S}_{b}\left(0,-\frac{1}{2} k_{y}, \omega\right)=2 \omega \mu_{0} Y_{0}^{-1} \tilde{J}\left(0,-\frac{1}{2} k_{y},-\gamma_{0}\left(0, \frac{1}{2} k_{y}, \omega\right)\right) \tilde{J}_{b}\left(0,-\frac{1}{2} k_{y},-\gamma_{0}\left(0, \frac{1}{2} k_{y}, \omega\right)\right)$ - $\sqrt{4 k_{1}^{2}(\omega)-k_{y}^{2}} /\left(\sqrt{4 k_{0}^{2}(\omega)-k_{y}^{2}}+\sqrt{4 k_{1}^{2}(\omega)-k_{y}^{2}}\right)^{2}$ where $\tilde{J}$ is the Fourier transform of the current density describing the normalized transmitting spectrum $\mathbf{S}$ in (5). 


\section{Inversion}

Using the fact that $\Delta \epsilon$ is real, this function can be found from its Fourier transform $\widetilde{\Delta \epsilon}$ through

$$
\Delta \epsilon(y, z)=\frac{1}{2 \pi^{2}} \operatorname{Re}\left[\iint_{k_{z} \geq 0} \widetilde{\Delta \epsilon}\left(k_{y}, k_{z}\right) \exp \left(i\left[k_{y} y+k_{z} z\right]\right) d k_{y} d k_{z}\right] .
$$

To use the relation between the measured data $s$ and $\widetilde{\Delta \epsilon}$ in (7) we must carry out the substitution $k_{z}=\sqrt{4 k_{1}^{2}(\omega)-k_{y}^{2}}$ and restrict $k_{y}$ to the range $\left|k_{y}\right|<2 k_{1}(\omega)$. Hence,

$$
\Delta \epsilon(y, z)=\frac{z}{2 \pi^{2}} \operatorname{Re}\left[\int_{0}^{\omega_{\max }} \int_{-2 k_{1}(\omega)}^{2 k_{1}(\omega)} \frac{4 k_{1}(\omega) \sqrt{\mu_{0} \epsilon_{1}} \tilde{s}\left(k_{y}, \omega\right)}{D\left(k_{y}, \omega\right) k_{z}} \exp \left(i\left[k_{y} y+k_{z} z\right]\right) d k_{y} d \omega\right]
$$

\section{Numerical Example}

The inversion scheme of Section 3 is now tested on synthetic GPR data. Figure 1 shows a two-layer dielectric pipe with outer diameter $24 \mathrm{~cm}$ and inner diameter $10 \mathrm{~cm}$ located $1 \mathrm{~m}$ below the interface. The permittivities of the soil, the inner and outer regions of the pipe are $10 \epsilon_{0}, 10.2 \epsilon_{0}$, and $10.4 \epsilon_{0}$, respectively. The synthetic GPR data are calculated using the method described in [4]. It is assumed that the radar uses 60 frequencies equally spaced in the range $20 \mathrm{MHz}<f<1.3 \mathrm{GHz}$. The antennas are assumed to be dipoles of length $2 \mathrm{~m}$, resistively loaded according to the Wu-King profile [5] to increase the bandwidth. Figures 2 and 3 show two images of $\Delta \epsilon(y, z) / \epsilon_{0}$ obtained from the inversion formula (10).

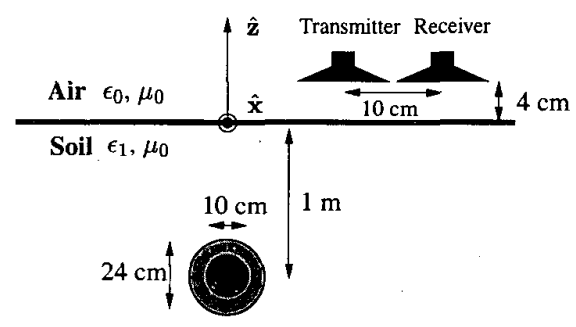

Figure 1: The fixed-offset GPR configuration involving a two-layer circular pipe. The antennas are resistively loaded $\hat{\mathbf{x}}$-directed dipoles.

In Figure 2 it is assumed that the antennas are Hertzian dipoles. It is seen that the image is highly distorted. In Figure 3 the correct antenna model is used. The current distribution on the antennas in the presence of the air-soil interface is determined using the method of moments. It's observed that the image is very accurate. This example shows that it's an inaccurate approximation to use simple Hertzian dipole models to describe realistic GPR antennas in diffraction tomography. 


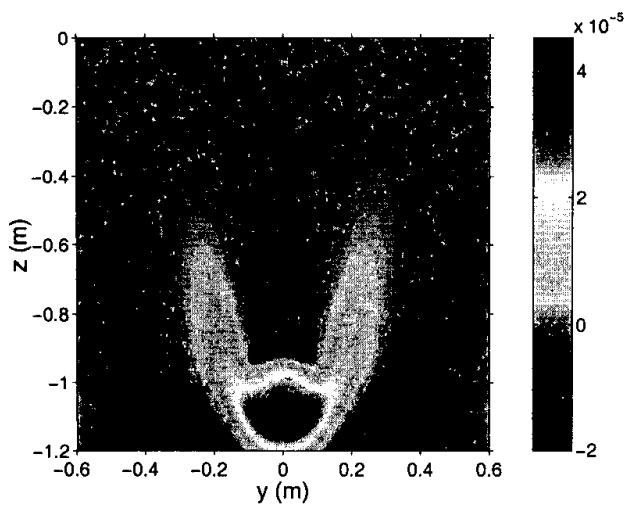

Figure 2: The image of $\Delta \epsilon(y, z) / \epsilon_{0}$. The antennas are modeled as Hertzian dipoles.

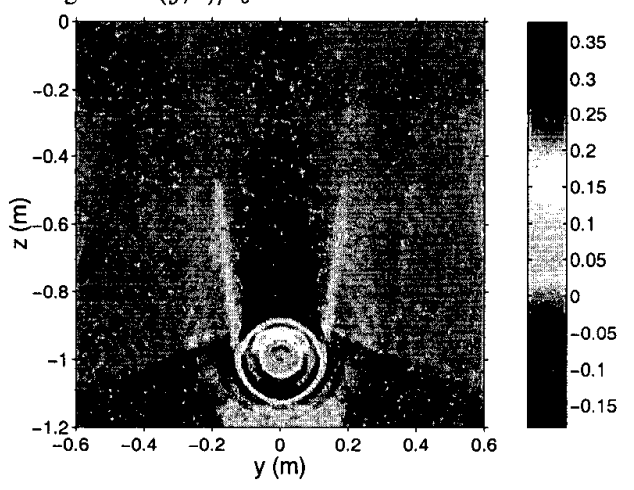

Figure 3: The antennas are modeled as resistively loaded dipoles.

\section{References}

[1] J. Molyneux and A. Witten, "Diffraction tomographic imaging in a monostatic measurement geometry," IEEE Trans. Geoscience and Remote Sensing, vol. 31, pp. 507511, Mar. 1993.

[2] T.B. Hansen and P. Meincke Johansen, "Inversion scheme for ground penetrating radar that takes into account the planar air-soil interface," IEEE Trans. Geoscience and Remote Sensing, vol. 38, pp. 496-506, Jan. 2000.

[3] P. Meincke, "Linear GPR inversion for lossy soil and a planar air-soil interface," IEEE Trans. Geoscience and Remote Sensing, vol. 39, pp. 2713-2721, Dec. 2001.

[4] T.B. Hansen and P. Meincke, "Scattering from a buried circular cylinder illuminated by a 3-D source," to be published in Radio Science.

[5] T.T. Wu and R.W.P. King, "The cylindrical antenna with nonreflecting resistive loading," IEEE Trans. Antennas and Propagation, vol. 13, pp. 369-373, May 1965. 